\title{
A Rare Case of an Internal Hernia of Small Bowel Masquerading as Pneumoperitoneum
}

\author{
Shireesh Gupta ${ }^{1}$, Prakash Alagarsamy², Ankit Goyal ${ }^{3}$
}

\begin{abstract}
Free gas under diaphragm is a very significant X-ray finding seen among patients of acute abdomen coming to the ER of any hospital. This finding usually suggests perforation of a hollow viscus and requires urgent surgical intervention. But there are some pathologies which mimic this important sign thereby necessitating the surgeon to always take a holistic view and approach any potential laparotomy with clinical suspicion. We have presented such a case seen in our emergency department wherein an internal herniation of bowel loops presented with pseudopneumoperitoneum.

Keywords: Chilaiditi syndrome, Internal herniation, Pseudopneumoperitoneum

Journal of Mahatma Gandhi University of Medical Sciences and Technology (2018): 10.5005/jp-journals-10057-0064
\end{abstract}

\section{INTRODUCTION}

Internal hernias occur as a result of protrusion of an internal organ into a foramen in the abdominal cavity. They are associated with pain, intestinal obstruction and gangrene. Pseudopneumoperitoneum describes any gas within the abdominal cavity that masquerades as free intraperitoneal gas or pneumoperitoneum when it is in fact contained within an organ. We present a case where internal herniation of small bowel lead to false diagnosis of pnemoperitoneum and laparotomy

\section{Case Description}

A fifty-year-old male patient presented to our emergency with the chief complaints of pain abdomen for the last 3 days which was insidious in onset, dull aching in character, progressive in nature and associated with vomiting and abdominal distension for 3 days, both of which are increasing in severity.

$O / E$ : Abdomen was tense, distended and tender. No organomegaly. Hernial sites were normal. Bowel sounds were sluggish. Patient was not passing flatus or stool.P/R: Fecal staining was present. No ballooning.

On nasogastric aspiration, semi-digested food was present.

Patient's chest X-ray showed air under diaphragm (Fig. 1).

USG showed minimal free fluid. Patient was dehydrated and had tachycardia and reduced blood pressure. After stabilizing the patient, decision was made to do an emergency laparotomy.

Intraoperative patient was found to have dilated bowel loops. A loop of small bowel was found to be present above the liver herniating through adhesions between liver and posterior surface of anterior abdominal wall (Fig. 2).

This internal hernia was causing obstruction leading to dilated bowel loops. Adhesions were removed. Bowel was reduced. No signs of gangrene were present. No perforated bowel loops were seen. Bowel was decompressed and abdomen closed in layers. Postoperative patient improved symptomatically.

\section{Discussion}

Air under the diaphragm (pneumoperitoneum) seen on plain radiograph usually signifies a perforated viscus. This usually
${ }^{1}$ Associate Professor, ${ }^{2,3}$ Junior Resident

${ }^{1-3}$ Department of General Surgery, Mahatma Gandhi University of Medical Sciences and Technology, Jaipur, Rajasthan, India

Corresponding Author: Shireesh Gupta, Associate Professor, Department of General Surgery, Mahatma Gandhi University of Medical Sciences and Technology, Jaipur, Rajasthan, India, e-mail: drshireeshgupta@rediffmail.com

How to cite this article: Gupta S, Alagarsamy P, Goyal A. A Rare Case of an Internal Hernia of Small Bowel Masquerading as Pneumoperitoneum. J Mahatma Gandhi Univ Med Sci Tech 2018;3(1):34-35.

Source of support: Nil

Conflict of interest: None

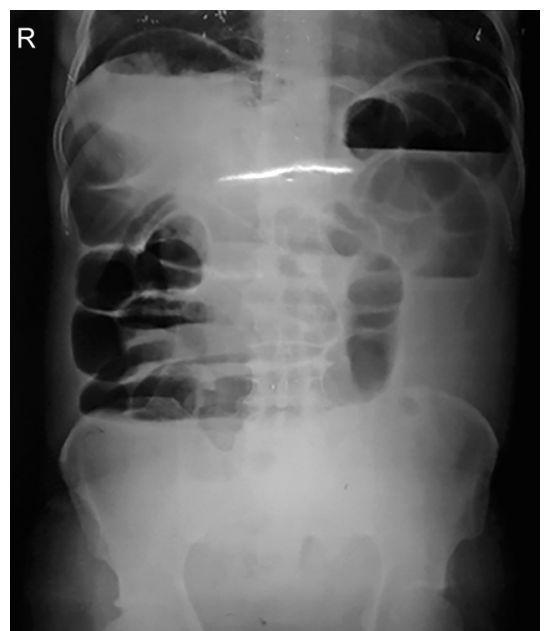

Fig. 1: Flat plate abdomen showing free gas under Diaphragm with multiple air fluid levels

represents a surgical emergency. However, not all air under the diaphragm seen on plain radiograph represents a surgical emergency.

Causes of pseudopneumoperitoneum include:

- Basal linear atelectasis

- Pneumomediastinum (mimicking cupola sign)

(อ) The Author(s). 2018 Open Access This article is distributed under the terms of the Creative Commons Attribution 4.0 International License (https://creativecommons. org/licenses/by-nc/4.0/), which permits unrestricted use, distribution, and non-commercial reproduction in any medium, provided you give appropriate credit to the original author(s) and the source, provide a link to the Creative Commons license, and indicate if changes were made. The Creative Commons Public Domain Dedication waiver (http://creativecommons.org/publicdomain/zero/1.0/) applies to the data made available in this article, unless otherwise stated. 


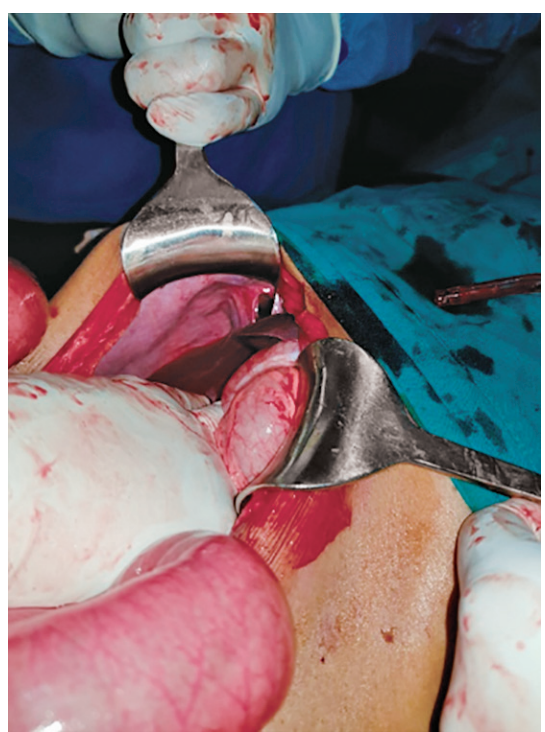

Fig. 2: Intraoperative picture showing internal herniation of small gut loops

- Chilaiditi syndrome

- Diaphragmatic undulation

- Gas within skin folds

- Biliary, portal vein or bowel wall gas

- Fat within the subdiaphragmatic space or the ligamentum teres
In our case, it was an internal hernia caused by adhesions masquerading as a pneumoperitoneum. A preoperative diagnosis was missed as the small bowel loops were involved, so no haustrations were present. Also the loops were grossly dilated leading us to make out only the bowel gas which lead us to make a diagnosis of pneumoperitoneum.

\section{Conclusion}

Internal hernia is a rare cause of intestinal obstruction. It is usually noted occurring through normal foramen or through mesentery. This case illustrates that pneumoperitoneum does not always means a case of perforation and other rare diagnosis such as chilaiditi syndrome or chronic intestinal pseudo-obstruction also should be kept in mind before deciding on laparotomy.

\section{References}

1. Melester T, Burt ME. Chilaiditi's syndrome. Report of three cases. JAMA. 1985;254:944-945.

2. Pseudopneumoperitoneum in chronic intestinal pseudo-obstruction: A case report. World J Gastroenterol 2011 Jun 28; 17(24): 2972-2975.

3. Ly JQ. The Rigler sign. Radiology. 2003;228:706-707

4. Mokrohisky JF. Pseudopneumoperitoneum; simulated free air in the peritoneal cavity. Am J Roentgenol Radium TherNucl Med. 1958;79:293-300.

5. Antonucci A, Fronzoni L, Cogliandro L, Cogliandro RF, Caputo C, De Giorgio R, Pallotti F, Barbara G, Corinaldesi R, Stanghellini V. Chronic intestinal pseudo-obstruction. World J Gastroenterol. 2008;14:29532961. 